\title{
A New Method of Parameter Estimation of Frequency-Hopping Signal
}

\author{
Yongli Qin, Ming Lv \\ Dept. of information engineering \\ University of Electronic Science and Technology of China \\ Chengdu, China \\ E-mail: yongliqinuestc@163.com; lvming@uestc.edu.cn
}

\begin{abstract}
Aiming at the resolution contradiction between time and frequency in frequency-hopping (FH) signal parameter estimation, a new FH signal parameter estimation method based on STFT is proposed by analyzing the influence of window function of STFT on the time-frequency spectrum. The parameter of FH signal can be extracted from the parameter of sliding window directly, avoiding the influence of time-frequency spectrum on the accuracy of estimation. The key point of this method is to seek the time-frequency spectrum which has the best time-frequency concentration and get the parameter of target sliding window by changing the beginning time and the width of the sliding window. Simulation results show the method is efficient to estimate the parameter of FH signal.
\end{abstract}

Keywords-parameter estimation; STFT; time-frequency spectrum; time-frequency concentration

\section{INTRODUCTION}

As one method of communication, the application of $\mathrm{FH}$ communication [1] in the field of military and civilian is obvious. Obtaining the enemy's parameter of FH signal in communication countermeasure is the prerequisite of intercepting or producing the optimal jamming. Timefrequency analysis is effective method to analyze FH signal. Bilinear transformation method has attracted the attention of many scholars with its high time-frequency resolution, but it brings a lot of trouble to the parameter estimation because of the existence of cross interference items. A lot of researches about cross-component suppression have been done, such as SPWVD. PWVD [2] [3] and so on. But all of these are at the cost of reducing the time-frequency resolution. Cohen and Affine suppress cross terms by smoothing the Wigner Ville, but there are a lot of remaining cross terms and some distribution may also introduce some new cross terms. In order to improve the performance of the distribution further, K.Kodera [4] put forward the idea of time-frequency rearrangement. Then F.A uger and P.F landrin [5] developed the rearrangement method. A lot have been achieved about cross-component suppression so far.

However, as the earliest method of time-frequency analysis, although STFT has no cross-component, it is restricted in the parameter estimation because of the resolution contradiction between time and frequency. So STFT is seldom used to estimate parameter directly. However, the step-size and width of the sliding window have an important influence on the time-frequency spectrum. Based on this point, parameters of FH signal can be extracted directly from the parameter of sliding window by studying the influence of the sliding window on the time-frequency spectrum of FH signal.

\section{SHORT-TIME FOURIER TRANSFORM}

Short-Time Fourier Transform (STFT) is one of the earliest methods of time-frequency analysis. Its discrete form is defined as [6]:

$$
\operatorname{STFT}_{X}(n, m)=\sum_{k=-\infty}^{k=+\infty} x(k) \omega^{*}(k T-n T) e^{-j 2 \pi(m F) k}
$$

The time-frequency resolution is influenced by the window function and it's impossible to obtain high time and frequency resolution at the same time because of the restriction of the uncertainty principle [7].

\section{PARAMETER ESTIMATION OF FH SIGNAL}

FH parameter estimation mainly includes the estimation of hop-timing, hop-rate and frequency-set. As the main method of FH parameter estimation, the time-frequency analysis is usually used to estimate parameter, but the timefrequency resolution often affects the accuracy of parameter estimation directly. As the most simple time-frequency analysis method, STFT can be used to estimate parameter as well and it has been used. The parameter can be extracted from time-frequency spectrum directly. Get difference after time-frequency analysis and then choose the threshold to remove interference peaks, get the distance between adjacent peaks, solve the data number in every hop-period, finally, and calculate the hop-rate by fs. The selection of the threshold is very import, if too large, it's easy to remove the useful information, and otherwise it can't get the purpose of denoising. The precision of the hop-rate estimation is affected by the marginal distribution of STFT which is not ideal, especially under low signal-to-noise ratio; In addition, the hop-timing is also affected by window function. All in all, most of the time-frequency analysis methods improve the accuracy of parameter estimation through improving the time-frequency resolution. Therefore, there are many algorithms about improving the precision of estimates, but a lot of algorithms are too complicated. Thinking form another angle, a new method can be found to solve this problem.

As a special kind of non-stationary signal, when each sliding window of STFT contains only one frequency, the time-frequency concentration is best, at this time, window function parameters are associated with $\mathrm{FH}$ parameter. So 
FH parameter can be estimated according to parameter of sliding window. Extracting parameter from the STFT function window parameters directly, it avoids the trouble caused by the defect of time-frequency analysis.This method is simple and clear.

Firstly, define spectrum peak proportion as D:

$$
D=\frac{\max \left(f f t_{x}\right)}{\sum_{i=1}^{i=N} f f t_{x}(i)}
$$

$\mathrm{fft}_{x}$ is the amplitude of $\mathrm{x}$ after STFT in a window function, $\max \left(f f t_{x}\right)$ is the maximum amplitude, $\sum_{i=1}^{i=N} f f t_{x}(i)$ is the sum of amplitude. In order to reduce the error, we take the average of $\mathrm{D}$ in multiple windows. The larger the spectrum peak proportion is, the better the timefrequency concentration is. Spectrum peak proportion is related to the performance of time-frequency concentration. Looking for the largest $\mathrm{D}$ is equivalent to looking for the best time-frequency concentration.

Sometimes, one or more frequency may be included in a function window of STFT. The amplitude-frequency graph represents the distribution of signal energy in frequency domain. The energy is mainly concentrated in one frequency when there is only one frequency in a sliding window and the $\mathrm{D}$ is the largest. When there are two frequencies in window, the $\mathrm{D}$ is changing with the change of proportion of two frequencies in the sliding window. When the proportion of two frequencies is equal, none of them plays a leading role and the $\mathrm{D}$ is smallest just as shown in figure 1. By that analogy, how the $\mathrm{D}$ will change can be known when there are multiple frequencies in a sliding window. When there is one frequency in the window, signal energy in the widow increases with the broadening of the window and is focused on the single frequency. The D increases step by step with the increasing of number of $\mathrm{f} 1$, just as shown in figure 2 .

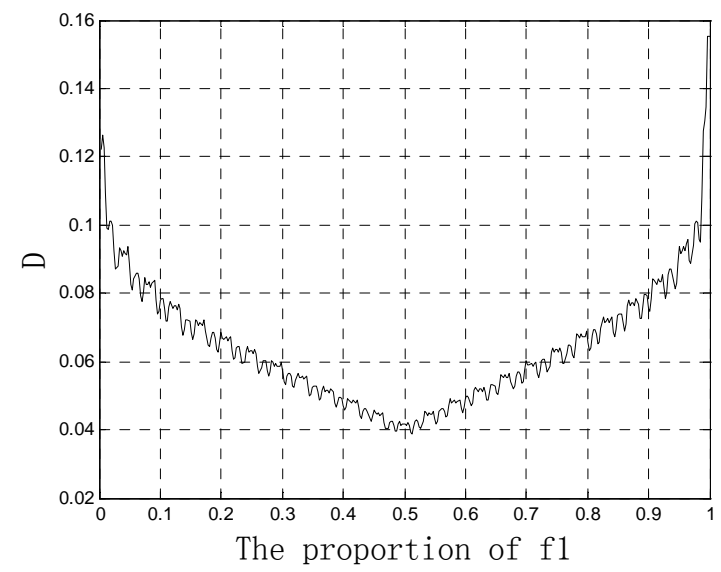

Figure 1. The relationship between $\mathrm{D}$ and proportion of $\mathrm{f} 1$

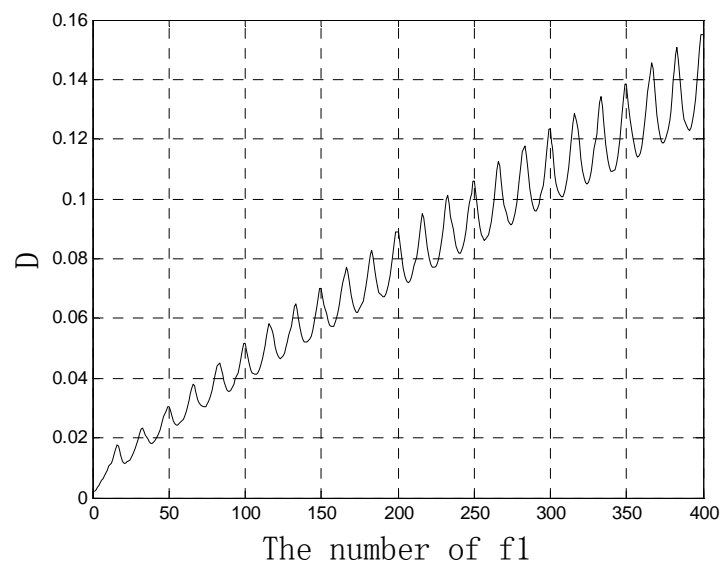

Figure2. The relationship between $\mathrm{D}$ and number of $\mathrm{f} 1$

\section{A. Hoping-Timing Estimation}

Firstly, assume that the width and step-size of sliding window are equal to the hop-period length, and then analyze the FH signal through STFT. If the $\omega(n)$ is the window function and the $x(n)$ is the FH signal, the STFT of $x(n)$ can be expressed as $\operatorname{STFT}_{x}(t, f)=f f t[x(n) \omega(n)]$.At this time, there is only one or two kinds of frequency in the window function. When only one frequency, there is only one peak in spectrum graph and the energy is concentrated in this frequency, so the $\mathrm{D}$ is biggest; otherwise, the $\mathrm{D}$ declines because of the dispersion of energy. The largest $\mathrm{D}$ can be found by changing the beginning time of sliding window. When the $\mathrm{D}$ is the largest, time-frequency concentration is the best. By now, the beginning time of sliding window is the frequency hop-timing.

\section{B. Hop-Rate Estimation}

Just like above section, assume the beginning time of window is equal to the hop-timing. The largest $\mathrm{D}$ can be found by changing the width of sliding window. The D increases with broadening of window when the width is less than the frequency hop-period. Figure 2 illustrates the principle. When the width of window is equal to the hopperiod, the D is largest. When more than period, the energy in the window begins to spread and the $\mathrm{D}$ begins to decline. So, when the D is largest, the width of sliding window is the hop-period and the inverse of window width is the hop-rate.

In fact, the estimation of hop-timing and hop-rate proceed at the same time. Look for the largest D by changing the beginning time and the width of sliding window. When the $\mathrm{D}$ is largest, the beginning time of window is the hoptiming and the inverse of the window width is the hop-rate. In order to reduce the times of searching, coarse parameter estimation is necessary. The approximate range can be got by traditional time-frequency analysis and then look for the goal window in this range. By this method, the parameters can be extracted from parameter of sliding window, avoiding influence of time-frequency concentration. 


\section{ALGORITHMS}

Step 1: load the FH data;

Step 2: signal sampling and the sampling rate is fs;

Step 3:coarse parameter estimation, determine the approximate extent;

Step 4: search the largest $\mathrm{D}$ in the approximate extent and determine the goal window function. Extract parameter from the sliding window.

\section{SIMULATION}

\section{A. Simulation parameter:}

$\begin{array}{ll}\text { Hop-rate: } & 1500 \mathrm{~h} / \mathrm{s} \\ \text { Sampling rate: } & 150 \mathrm{kHz} \\ \text { Hop-timing: } & 40 / 1500(\mathrm{~s}) \\ \text { Frequency-set: } & 4 \mathrm{kHz}-30 \mathrm{kHz} \\ \text { SNR: } & -5 \mathrm{~dB}-30 \mathrm{~d}\end{array}$

\section{B. Hop-Timing and Top-Rate Estimation}

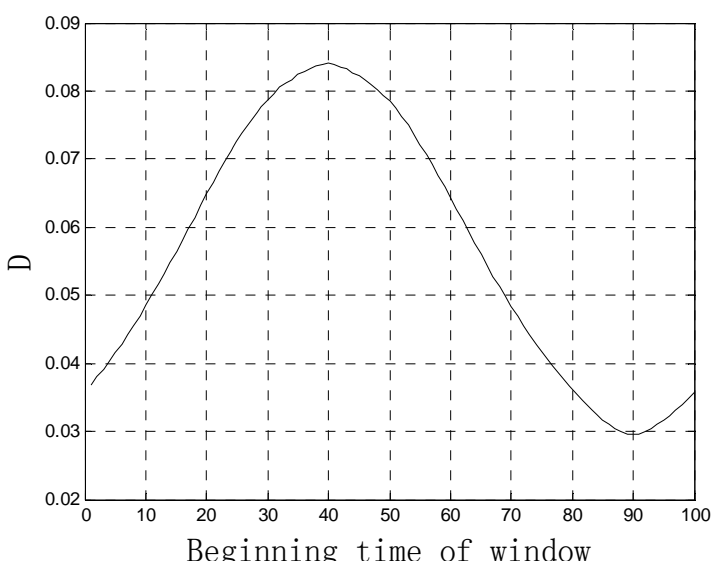

Figure 3. Relationship between $\mathrm{D}$ and beginning of sliding window

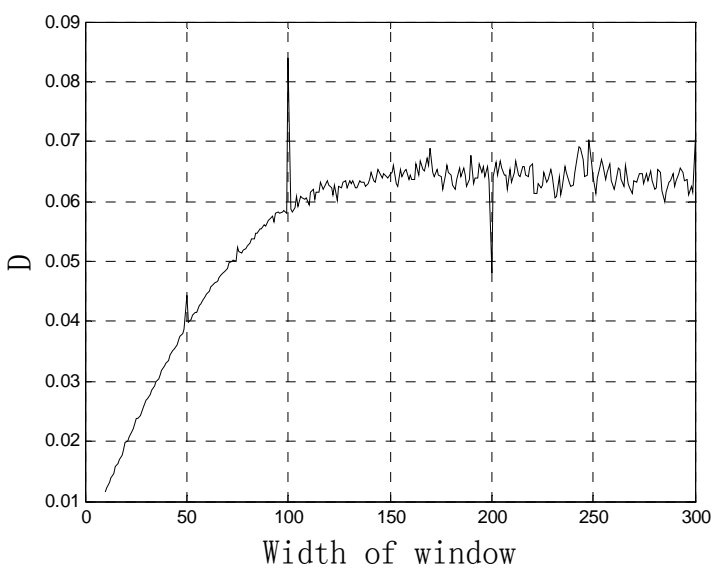

Figure 4. Relationship between $\mathrm{D}$ and width of sliding window
Figure 3 is the simulation diagram of hop-timing estimation. The first peak appears at 40, so the hop-timing is $40 /$ fs and it coincides with the assumption. Figure 4 is the simulation diagram of hop-rate estimation. the maximum appears near 100 , it means the hop-period is 100 , so the hoprate is about $1500 \mathrm{~h} / \mathrm{s}$ (fs/100). It's also consistent with the simulation conditions.

\section{Estimation on Different SNR}

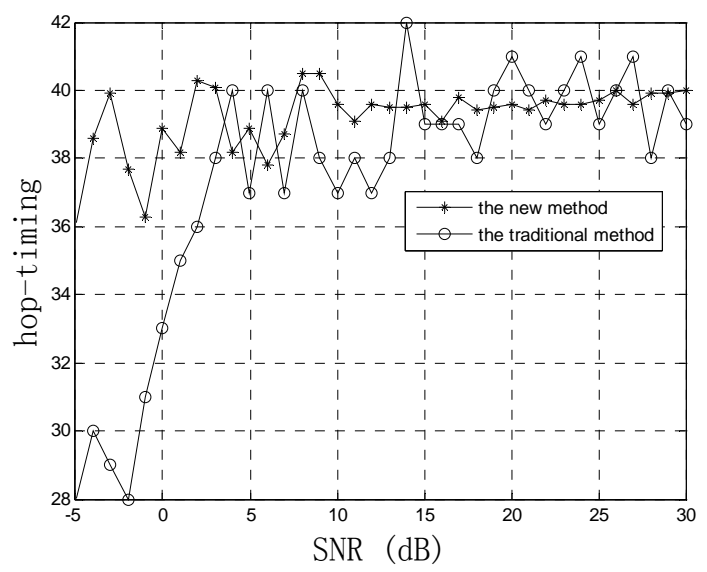

Figure 5. Hop-timing estimation on different SNR

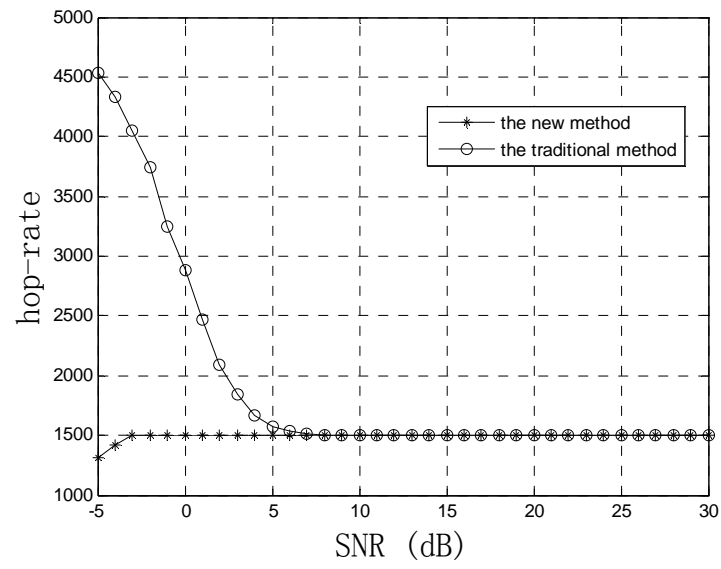

Figure 6. Hop-rate estimation on different SNR

Figure 5 and Figure 6 are the simulation diagrams of hop-timing and hop-rate on different SNR in different method. One method is time-frequency analysis and the other is the method mentioned in this paper. As can be seen from figure 5, the method is superior to the other, especially in low signal-to-noise ratio. Figure 6 shows the method is superior to the other in low signal-to-noise ratio.

\section{CONCLUSIONS}

In this paper, a new method is introduced .The parameter of FH signals can be extracted from the parameter of the sliding window directly. There is no need to consider the effect of time-frequency concentration on parameter estimation precision. The simulation results show that the 
new method for parameter estimation of FH signal based on STFT is efficient.

\section{REFERENCES}

[1] Mei Wen-hua, Wang Shu-bo, Qiu Yong-hong, Frequency hopping communication, Beijing: National Defence Industry Press, 2005, 89.(In Chinese)

[2] Barbarossa S, Scaglione A. Parameter Estimation of Spread Spectrum Frequency-hopping Signals using Time-Frequency Distributions[C]//First IEEE Signal Processing Workshop on Signal Processing Advances in Wireless.
[3] Zhao Jun, Zhang Zhao-yang, Lai Li-feng. Blind Parameter Estimation of Frequency-hopping Signals Based On Time-Frequency Analysis [J]. Journal of circuits and systems, 2003, 8(3): 46-50.

[4] K. Kodera, R. Gendrin, C. D. Villedary. Analysis of time-varying signals with small BT values. IEEE Transaction on Acoustic, Speech, and, Signal Processing, 1978, 26(1):64-76.

[5] F. Auger, P. Flandrin. Improving the readability of time-frequency and time-scale representations by the reassignment method. IEEE Trans. Signal Processing,1995, 43(5): 1068-1089.

[6] Wang Xue-gang, Zhang Ming-you. Modern signal theory, Beijing: Publishing House of Electronics Industry, 2005, 162-200.

[7] Hlawatsch F, Auger F. Time-Frequency Analysis: Concepts and Methods [M]. London (UK): ISTE and Wiley, 2008. 